\title{
A novel approach to measure photodesorption rates of interstellar ice analogues The photodesorption rate of $\mathrm{CO}$ ice reinvestigated
}

\author{
D. M. Paardekooper, G. Fedoseev ${ }^{\star}$, A. Riedo, and H. Linnartz
}

\begin{abstract}
Raymond and Beverly Sackler Laboratory for Astrophysics, Leiden Observatory, Leiden University, PO Box 9513, 2300 RA Leiden, The Netherlands

e-mail: dmpaardekooper@strw.leidenuniv.nl
\end{abstract}

Received 6 June 2016 / Accepted 28 June 2016

\begin{abstract}
Context. In recent years photodesorption rates have been determined in dedicated laboratory experiments for a number of different interstellar ice analogues. These rates are important in order to model non-thermal desorption processes that, for example, affect gas-phase abundances of species and determine the position of photo-induced snow lines in protoplanetary disks. However, different groups using similar experiments have found significant deviating photodesorption values.

Aims. Here a new measurement concept is introduced that allows photodesorption rates to be determined following a different experimental approach. The potential of this method is demonstrated using the example of pure CO ice, the solid that gives the most striking discrepancies in the published results.

Methods. The new experimental approach uses laser desorption post-ionisation time-of-flight mass spectrometry. It is based on the concept that the physical and geometrical properties of the plume obtained by laser induced desorption of the ice directly depend on the original ice thickness. This allows the ice loss to be determined as function of vacuum ultraviolet (VUV) fluence, which results in a photodesorption rate. The method has the additional advantage that it records all ice species, including photoproducts generated by the VUV irradiation. As a consequence, the method introduced here is also suited to determine the overall photodesorption rate of mixed ices.
\end{abstract}

Results. The photodesorption rate for $\mathrm{CO}$ ice at $20 \mathrm{~K}$ has been determined as $(1.4 \pm 0.7) \times 10^{-3}$ molecules per incident VUV photon This result is compared to existing experimental and theoretical values and the astronomical relevance is discussed.

Key words. ultraviolet: ISM - methods: laboratory: solid state - ISM: molecules - ISM: abundances - molecular processes astrochemistry

\section{Introduction}

Astronomical surveys reveal the presence of gas phase molecules in dense and cold regions of the interstellar medium (ISM), where these molecules are expected to be frozen out on top of (sub-)micron sized dust grains (Piétu et al. 2007; Caselli et al. 2012; Vastel et al. 2014). This observation indicates that an ongoing process must exist that allows these species to form in the gas phase or that continuously replenishes them from a solid state reservoir. The latter is more likely, as for $\mathrm{CH}_{3} \mathrm{OH}$, for example, only efficient surface formation routes are known (Watanabe \& Kouchi 2002; Fuchs et al. 2009; Linnartz et al. 2015). Clearly, non-thermal desorption processes are at play. Various mechanisms have been proposed including cosmic ray spot heating of the grains (Leger et al. 1985); chemical desorption, i.e. desorption due to excess energy of a surface reaction (Dulieu et al. 2013; Chang \& Herbst 2016); and vacuum ultraviolet (VUV) induced photodesorption. Particularly, photoinduced desorption processes have attracted considerable interest, starting in 1995 with the experimental measurement of the photodesorption rate of water ice (Westley et al. 1995a,b) and

\footnotetext{
* Present address: INAF-Osservatorio Astrofisico di Catania, via Santa Sofia 78, 95123 Catania, Italy.
}

more recently (since 2007) for a large number of other ices (an overview can be found in e.g. Ioppolo et al. 2014).

In cold and dense molecular clouds, the interstellar radiation field is strongly attenuated due to shielding by dust grains, but penetrating cosmic rays can excite $\mathrm{H}_{2}$ and the resulting emission typically peaks at Ly- $\alpha$ wavelengths (Prasad \& Tarafdar 1983; Gredel et al. 1989; Shen et al. 2004). Molecules condensed on top of cold dust grains or, alternatively, formed in the ice layers can undergo photodesorption following VUV excitation, transferring solid state species into the gas phase. Later on in the star and planet formation sequence, light from the young stellar object also becomes important, determining for example the position of photo-induced snowlines (Öberg et al. 2015). Therefore, accurate photodesorption rates are needed to quantify the role that (inter)stellar radiation has on abundances in the solid state and gas phase in different astronomical environments, varying from molecular clouds (Hollenbach et al. 2009) to protoplanetary disks (Willacy \& Langer 2000; Drozdovskaya et al. 2014; Walsh et al. 2015).

A large number of extensive laboratory studies has been reported on the photodesorption behaviour of interstellar ices. In particular, pure carbon monoxide (CO) ice has been in the spotlight. The low accretion temperature of $\mathrm{CO}$ results in a coating on top of the ice-covered dust grain. 
Using a $\mathrm{H}_{2}$ microwave discharge lamp emitting at Ly- $\alpha$ and around $160 \mathrm{~nm}$, Öberg et al. (2007, 2009b) studied the (nondissociative) photodesorption of $\mathrm{CO}$ ice. The resulting value, $(2.7 \pm 1.3) \times 10^{-3}$ molecules photon ${ }^{-1}$ measured at $15 \mathrm{~K}$, was much higher than used at that time in astrochemical models (Draine \& Salpeter 1979; Hartquist \& Williams 1990). Similar experiments by other groups (Muñoz Caro et al. 2010; Chen et al. 2014; Muñoz Caro et al. 2016) found deviating and even higher values in the $10^{-2}$ to $10^{-1}$ molecules per photon range. A possible explanation for these deviations was searched for in the spectral emission pattern of the used broad-band light sources that turned out to be more sensitive on the parameter settings than assumed (Chen et al. 2014; Es-sebbar et al. 2015; Ligterink et al. 2015). This assumption was in line with wavelength dependent $\mathrm{CO}$ photodesorption rates recorded at the DESIRS beamline of the SOLEIL synchrotron facility (Fayolle et al. 2011) that showed that the photodesorption of CO follows a wavelength dependent desorption induced by electronic transition (DIET) mechanism that is found to be more effective around $160 \mathrm{~nm}$ than at Ly- $\alpha$. The CO photodesorption rate furthermore strongly depends on the deposition temperature, as has been discussed by several groups (Öberg et al. 2009b; Muñoz Caro et al. 2010, 2016). In parallel, many more studies have been performed on other pure ices, including $\mathrm{H}_{2} \mathrm{O}, \mathrm{N}_{2}, \mathrm{CO}_{2}, \mathrm{O}_{2}\left(\mathrm{O}_{3}\right)$, and $\mathrm{CH}_{3} \mathrm{OH}$, as well as a few mixed ices, $\mathrm{CO}: \mathrm{N}_{2}, \mathrm{CO}: \mathrm{H}_{2} \mathrm{O}$, and $\mathrm{CO}: \mathrm{CH}_{3} \mathrm{OH}$ (Öberg et al. 2009a; Hama et al. 2010; Chen et al. 2011; Bahr \& Baragiola 2012; Bertin et al. 2012, 2013, 2016; Fayolle et al. 2013; Yuan \& Yates 2013; Fillion et al. 2014; Zhen \& Linnartz 2014; Martín-Doménech et al. 2015). In many of these studies it became clear that upon VUV photolysis, molecules not only photodesorb, but may also be involved in photo-induced reactions (Öberg 2016) substantially complicating the analysis, as photoproducts may photodesorb as well (see e.g. the discussion on $\mathrm{O}_{2}$ and $\mathrm{O}_{3}$ photodesorption upon VUV irradiation of $\mathrm{O}_{2}$ ice in Zhen \& Linnartz 2014). A number of theoretical studies (Galloway \& Herbst 1994; Dzegilenko et al. 1995; Andersson et al. 2006; Andersson \& van Dishoeck 2008; Arasa et al. 2010, 2011, 2015; van Hemert et al. 2015) have focused on the molecular processes at play, confirming the high level of complexity involved.

In the majority of the above-mentioned laboratory studies, photodesorption rates have been obtained using two different methods, based on IR (transmission or reflection) spectroscopy or mass spectrometry (Ioppolo et al. 2014). In the first case a decreasing IR signal reflects the loss in surface abundance of molecules that is monitored as a function of VUV fluence, while in the second case the gas-phase abundance of the desorbing species is measured. Both methods allow quantitative values to be derived because both IR and mass signals can be linked to absolute molecule numbers. Infrared spectroscopy only allows the monitoring of polar species. A decreasing IR signal with time reflects the loss rate of a specific molecule upon irradiation, but does not differentiate between a signal decrease because of a direct photodesorption process or because of a decrease in the precursor abundance due to a photochemical process. In this case photodepletion is a better way of describing the processes taking place. Moreover, for larger molecules spectral congestion and spectral overlaps cause additional problems. Mass spectrometry overcomes many of these problems; however, the conversion between gas phase mass signals and absolute photodesorption rates is challenging. In the present study, the photodesorption of pure $\mathrm{CO}$ ice is reinvestigated using a new experimental concept. By means of laser desorption, complete desorption of the ice is realised at the laser spot. The resulting desorption plume is characterised for a series of different extraction times using electron impact ionisation in conjunction with time-of-flight mass spectrometry. The absolute intensity of specific mass to charge $(\mathrm{m} / \mathrm{z})$ signals provides information on the thickness of the ice at a specific time (i.e. for a specific fluence), while each time-of-flight spectrum contains information of the possible photoproducts. This measurement concept is explained in detail in the next section. The applications are illustrated in Sect. 3 and are discussed in Sect. 4, along with their astronomical relevance.

\section{Experimental methods}

\subsection{Experimental set-up}

The experiments have been carried out in our ultra-high vacuum (UHV) set-up MATRI ${ }^{2}$ CES, described in detail in a previous publication (Paardekooper et al. 2014). Briefly, MATRI ${ }^{2}$ CES consists of two different UHV chambers, a main chamber and a time-of-flight chamber. The base pressure of these chambers is in the $10^{-10}$ mbar range. The main chamber hosts a gold-coated $\mathrm{Cu}$ substrate in thermal contact with a closed-cycle He cryostat, enabling substrate temperatures down to $20 \mathrm{~K}$. The absolute temperature accuracy is better than $\pm 1 \mathrm{~K}$, while the relative precision is $\pm 0.25 \mathrm{~K}$. The cryostat is mounted on top of two translators $(x, z)$ which enable us to systematically probe different surface spots. The vertical $(z)$ translation stage is fully motorised. Control of substrate temperature is achieved using a resistive heater element, a thermocouple and a Lakeshore temperature regulator. Ices can be grown on top of the cold substrate by leaking in gas-phase species through an all-metal needle valve attached to a capillary. $\mathrm{CO}$ gas has been used without further purification (CO, 4.7 Praxair).

The deposition rate of the individual species is determined by $\mathrm{HeNe}$ laser interference measurements. Details of this procedure are provided in Sect. 2.2. Photodesorption of the ice is induced using VUV photons produced by a microwave $\mathrm{H}_{2}$ discharge lamp. The lamp (borosilicate) has a F-type design (Ligterink et al. 2015), and is evacuated using a scroll pump. The plasma is powered by a Sairem microwave generator using an Evenson cavity. The plasma is ignited using a high frequency generator. Typical settings amount to 0.85 mbar of $\mathrm{H}_{2}$ and $80 \mathrm{~W}$ of applied microwave power. This lamp directly faces the ice sample through an UHV compatible $\mathrm{MgF}_{2}$ window that is positioned $14 \mathrm{~cm}$ from the substrate. The VUV flux calibration procedure is described in Sect. 2.3.

The used detection scheme is based on laser desorption postionisation time-of-flight mass spectrometry (LDPI TOF-MS). The desorption of the ice sample is induced using the unfocused, skimmed beam $(\sim 1 \mathrm{~mm})$ of the third harmonic of a $\mathrm{Nd}$ :YAG laser (355 nm, 4-5 ns). The pulse power of the laser is reduced using an attenuator, resulting in a typical laser pulse energy of $\sim 35 \mathrm{~mJ} \mathrm{~cm}^{-2}$, ensuring a localised and complete desorption of the ice only at the impacting spot. Subsequently, the desorbed species are ionised by electrons with a mean energy of $70 \mathrm{eV}$ using an electron impact ionisation source. The generated ions are extracted by ion optics, situated in close vicinity of the cold substrate and directed into the time-of-flight chamber. The ions drift and are separated based on their mass to charge $(\mathrm{m} / \mathrm{z})$ ratios, after which they are detected by a Micro Channel Plate (MCP) detector. The resulting signal is then digitised by a data acquisition (DAQ) card.

Complete desorption of the ice at the laser spot is needed to guarantee that the desorption plume contains all the information 
about the original ice thickness of the sample. The detailed characterisation of the plume structure is performed in the following way. After each laser pulse, the extraction grid is triggered sequentially every $20 \mu \mathrm{s}$ (pulse width $=1 \mu \mathrm{s}$ ) and a single timeof-flight spectrum is recorded. This single spectrum contains information of different parts of the desorption plume. By changing the relative timing between the first extraction trigger and the laser pulse from $0,5,10$, to $15 \mu \mathrm{s}$, the complete plume structure with $5 \mu$ s resolution can be unravelled. The timing sequence is obtained using two coupled pulse generators (DG535, Stanford Research Systems) running at $10 \mathrm{~Hz}$ and $50 \mathrm{kHz}$. The lower frequency triggers the laser (flash lamp and Q-switch) and the acquisition of the DAQ card. The higher frequency triggers the extraction grid multiple times during every cycle of the lower frequency pulse generator. In parallel, the vertical translator moves down with constant velocity, while different spots of the sample are hit by laser pulses inducing desorption. A total of 44 time-of-flight traces are obtained for each extraction time $(0,5,10$, and $15 \mu \mathrm{s})$. Next to the multi-extraction trigger TOF spectrum described above, TOF spectra with enhanced signal-to-noise ratio $(\mathrm{S} / \mathrm{N})$ can be obtained using a single extraction trigger. A custom-made LabVIEW routine controls the full experimental procedure including the data acquisition.

\subsection{Deposition rate calibration}

The deposition rate of $\mathrm{CO}$ has been determined using $\mathrm{HeNe}$ laser interference measurements (Baratta \& Palumbo 1998; Bossa et al. 2015). An intensity stabilised HeNe-laser (Thorlabs HRS015) is used as a light source. The laser beam is polarised by means of a transmissive s-polariser and aimed at the sample with a $1.19^{\circ}\left(\theta_{0}\right)$ incident angle. The reflected beam is recorded by a photodiode (Thorlabs PDA36A), using an oscilloscope to digitise the signal (Tektronix 2022B). A custom-made LabVIEW program is used to record both time and photodiode signal.

The deposition rate $\left(\Gamma\right.$ in molecules $\mathrm{cm}^{-2} \mathrm{~s}^{-1}$ ) is determined by combining Eqs. (1) and (2) (Bouilloud et al. 2015).

$$
\begin{aligned}
& d=\frac{\lambda}{2 n_{1} / n_{0} \cdot \cos \left(\theta_{f}\right)} \\
& \Gamma=\frac{d \cdot \rho \cdot N_{a}}{M \cdot t},
\end{aligned}
$$

where $d$ corresponds to the thickness increase during subsequent interference maxima in $\mathrm{nm}, \lambda$ is the wavelength of the laser $(632.8 \mathrm{~nm}), n_{0}$ and $n_{1}$ are the refractive indices of vacuum and ice, $\theta_{f}$ is the angle of refraction in the ice in degree, $\rho$ is the density in $\mathrm{g} \mathrm{cm}^{-3}, N_{\mathrm{a}}$ is Avogradro's constant $\left(6.022 \times 10^{23} \mathrm{~mol}^{-1}\right)$, $M$ is the molar mass of the species, and $t$ is the time in seconds.

In this way, for carbon monoxide ice growing at $20 \mathrm{~K}$, a deposition rate is obtained of $1.7 \times 10^{13}$ molecules $\mathrm{cm}^{-2} \mathrm{~s}^{-1}$ using a density of $0.80 \mathrm{~g} \mathrm{~cm}^{-3}$ and $n_{1}=1.27$ (Roux et al. 1980). It is important to note that throughout this article the ice thickness has been converted to the surface coverage, in molecules $\mathrm{cm}^{-2}$, which has the same dimensions as a column density.

\subsection{Photon flux calibration}

As stated before $\mathrm{CO}$ has different photodesorption rates for different wavelengths and therefore it is important to know the spectral energy distribution (SED) characteristics of the lamp. Therefore, the SED of the microwave powered $\mathrm{H}_{2}$-discharge lamp is used as characterised recently, using an absolute calibrated VUV spectrometer (Ligterink et al. 2015). The resulting spectrum has been presented in Paardekooper et al. (2016). The emission profile predominately contains photons at Ly- $\alpha$ and also has contributions from molecular hydrogen at $160 \mathrm{~nm}$. The photon flux at sample distance has been measured using a $1 \mathrm{~cm}^{2}$ National Institute of Standards and Technology (NIST) calibrated AXUV-100 photodiode. The photocurrent is determined with a Keithley 485 picoammeter. It should be noted that not only the Ly- $\alpha$ and $160 \mathrm{~nm}$ emissions, but also visible photons can contribute to the observed photocurrent. In order to deconvolute the contribution of the latter, the emission of a sealed plasma lamp has been measured with the same plasma conditions. The sealed plasma lamp absorbs all VUV photons $(\lambda<300 \mathrm{~nm})$, and has a transmittance of $(90 \pm 3) \%$ in the visible regime.

Both the SED of the discharge lamp and quantum efficiencies at different wavelengths of the NIST photodiode are known. Therefore, the effective quantum efficiency can be determined. The VUV photon flux is then derived as:

$$
F(\lambda)=\frac{i(\lambda)}{e \cdot \epsilon(\lambda)}
$$

with $i(\lambda)$ the photocurrent (resulting from VUV photons) in Ampere, $e$ the electron charge $\left(1.602 \times 10^{-19} \mathrm{C}\right)$, and $\epsilon(\lambda)$ the quantum efficiency, i.e. electron per exposed photon. At sample location, the VUV flux for the settings given before, is determined to be $(2.3 \pm 0.4) \times 10^{14}$ photons $\mathrm{cm}^{-2} \mathrm{~s}^{-1}$.

\subsection{Detection scheme}

The plume structures are recorded using a multi-trigger timeof-flight scheme for different surface coverages (e.g. different deposition times). These calibration measurements allow us to directly link plume structures to the surface coverages. In the case of $\mathrm{CO}$, the plume structures have been recorded for surface coverage, ranging from $4 \times 10^{15}$ to $8 \times 10^{15} \mathrm{CO}$ molecules $\mathrm{cm}^{-2}$.

Subsequently, a fresh ice with a surface coverage of $8 \times$ $10^{15} \mathrm{CO}$ molecules $\mathrm{cm}^{-2}$ ice is prepared and then processed using VUV photons produced by the $\mathrm{H}_{2}$ discharge lamp. The VUV photons induce desorption following a constant parameter defined by the photodesorption rate. The plume structure resulting upon laser desorption is measured after different times, i.e. at different VUV fluence, from which the surface coverage can be unravelled. After photo-processing the ice, different column positions can be selected by shifting the horizontal translator $(x)$. Between subsequent measurements, the sample is returned to the reference position in order to guarantee full overlap between VUV photons and the ice sample. In an area where the ice growth is homogenous, four columns are selected for plume profile characterisation.

Combined with the VUV photon flux measurement, this allows the quantitative determination of the photodepletion rate. At this stage it is important to discriminate explicitly between photodesorption and photodepletion. Photodesorption is defined as the mechanism where molecules in the ice evaporate (in)directly upon absorption of a VUV photon, whereas photodepletion also takes into account the effect of photochemistry. In the case of $\mathrm{CO}$ the role of photochemistry will be limited, given the high binding energy. Even though negligible on the final photodesorption value, the process can be visualised with our new approach, as will be discussed later. Compared to regular photodesorption studies using IR spectroscopy and quadrupole mass spectrometry, this approach has the additional advantage 


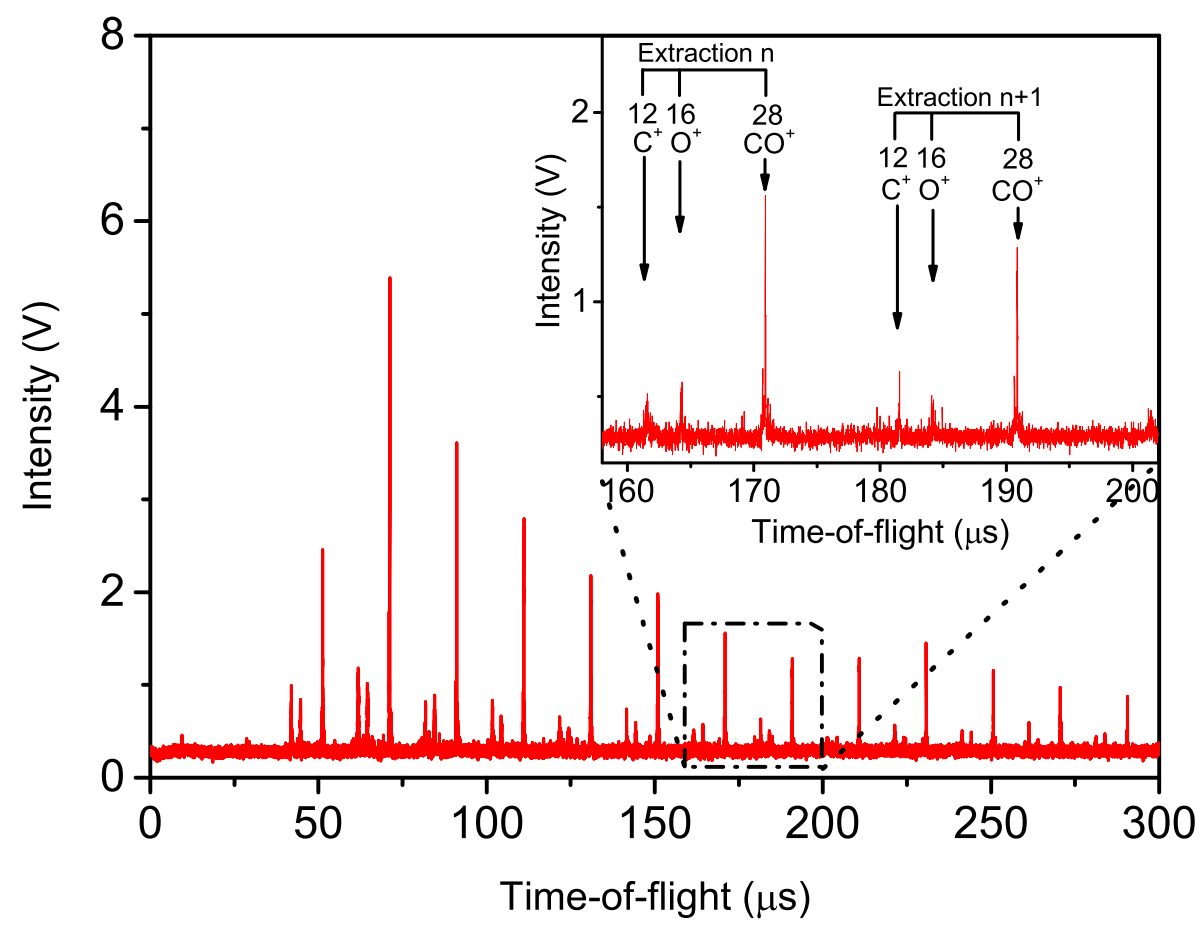

Fig. 1. Time-of-flight spectrum of laser induced desorbed CO ice $\left(8 \times 10^{15}\right.$ molecules $\left.\mathrm{cm}^{-2}\right)$ using multiple extraction pulses with a spacing of $20 \mu \mathrm{s}$. The inset shows two sequential extractions including the mass calibration. This spectrum is the sum of 44 individual spectra.

that all ice constituents present in the ice can be traced. The plume structure contains direct information of the surface coverage of the ice, while at the same time other products resulting from photochemistry are detected. This provides a generally applicable method to determine the photodesorption rate of mixed ices as well. This is not that relevant for $\mathrm{CO}$, as pure $\mathrm{CO}$ ice is expected to exist in interstellar ices, until it gets mixed with hydrogenation products such as $\mathrm{H}_{2} \mathrm{CO}$ and $\mathrm{CH}_{3} \mathrm{OH}$ (Fuchs et al. 2009; Cuppen et al. 2010). Many other species are expected to exist only in intimately mixed geometries. In this respect, the present technique is different to the previously used RAIRS; it has the advantage that all species formed in the ice (including species invisible for RAIRS) can be monitored in the diagnostic step, i.e. the plume with desorbed species contains not only the precursor, but also those species formed upon VUV irradiation. This does not provide direct information on the desorbing species, but it characterises the initial and final species present in the ice.

\section{Results}

\subsection{Plume characterisation of laser desorbed CO ice}

Figure 1 shows a typical time-of-flight trace obtained using multiple extraction pulses of $\mathrm{CO}$ ice with a substrate coverage of $8 \times 10^{15}$ molecules $\mathrm{cm}^{-2}$. Compared to previous measurements on $\mathrm{MATRI}^{2} \mathrm{CES}$ the $\mathrm{S} / \mathrm{N}$ for these spectra is lower; this is a direct result of the increased number of extraction pulses per time-of-flight spectrum combined with fewer averages per spectrum. Since the number of extraction pulses has increased, more ions are allowed to enter the time-of-flight chamber, leading to a higher noise level. The desorbed species are ionised using the electron impact ionisation source, which leads to both ionisation and dissociation of the species. In the case of $\mathrm{CO}$, the number of resulting species is limited and this results in $\mathrm{CO}^{+}, \mathrm{C}^{+}$, and $\mathrm{O}^{+}$. As shown in the inset of Fig. 1, all these ions are observed within each individual extraction pulse.

By changing the relative timing of the laser and the extraction pulse, the complete desorption profile of the plume can

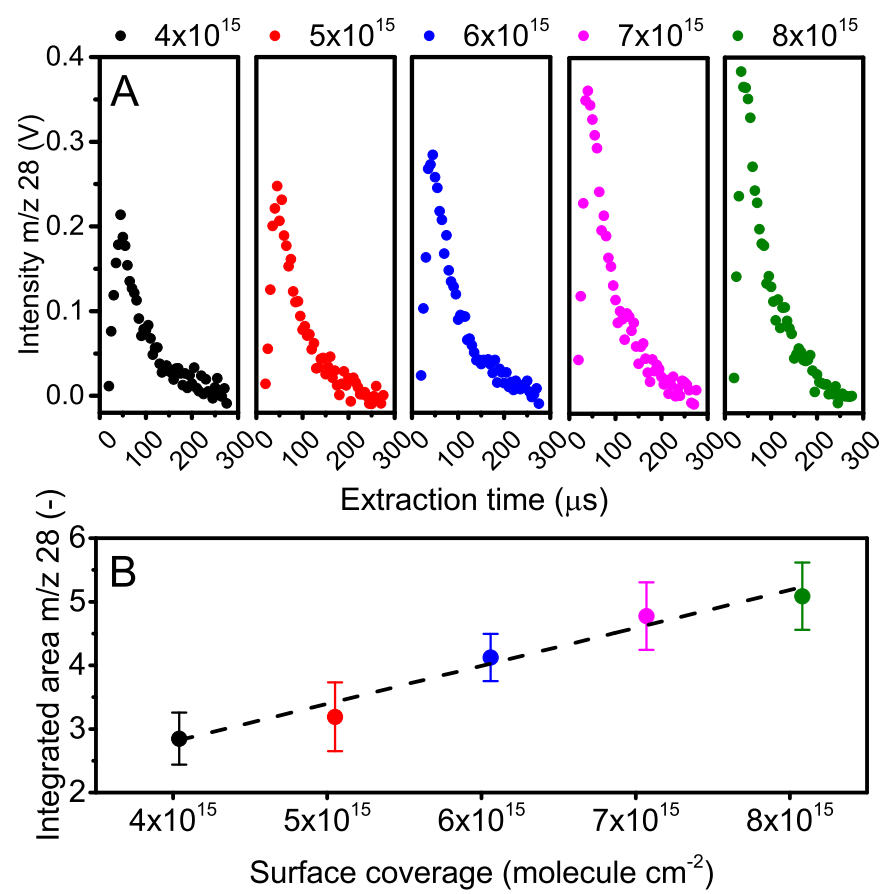

Fig. 2. Upper panel A): plume profiles observed for $m / z=28$ for different $\mathrm{CO}$ substrate coverage ranging from $4 \times 10^{15}$ molecules $\mathrm{cm}^{-2}$ to $8 \times$ $10^{15}$ molecules $\mathrm{cm}^{-2}$ upon laser induced desorption. Lower panel $\left.\mathbf{B}\right)$ : integrated area of $m / z=28$ of the complete plume profiles for the corresponding surface coverage (same colour coding).

be characterised. In Fig. 2A the plume profiles for different $\mathrm{CO}$ substrate coverages are depicted. The plume profiles are described well using a time dependent Boltzmann equation:

$S\left(t, T_{\text {trans }}\right)=A \cdot t^{-4} \cdot \mathrm{e}^{\frac{-m \cdot r^{2}}{2 \cdot k_{\mathrm{b}} \cdot t^{2}}}$

Here $A$ is a scaling factor, $T_{\text {trans }}$ the translational energy, $t$ the time, $m$ the mass of the molecule, $r$ the distance travelled, and $k_{\mathrm{b}}$ the Boltzmann constant (DeSimone et al. 2013). 


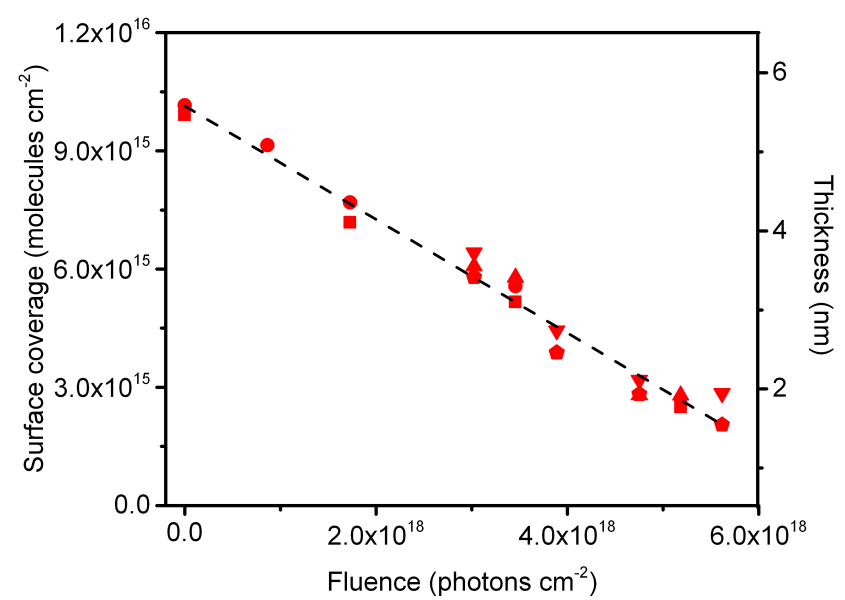

Fig. 3. Substrate coverage of CO (left axis), and thickness of the CO ice (right axis) as a function of different VUV fluence. The results for five experiments, starting from different initial thicknesses are shown, and for clarity have been horizontally shifted around the fitted line that is the resulting average of the photodesorption rates of these independent measurements. The photodepletion of $\mathrm{CO}$ ice at $20 \mathrm{~K}$ can be determined by a linear fit. Surface coverage has been determined with Eqs. (1) and (2), with $n=1.27$ and $\rho=0.80 \mathrm{~g} \mathrm{~cm}^{-3}$.

Assuming that most ions originate from the centre of the extraction optics $( \pm 2.5 \mathrm{~mm})$, the translation energy ranges between $(2 \pm 1) \times 10^{2} \mathrm{~K}$.

Using the calibration of the plume profiles for different values of $\mathrm{CO}$ surface coverage presented in Fig. 2B, it is now possible to determine the remaining surface coverage of $\mathrm{CO}$ ice for different VUV fluences.

\subsection{Photodesorption of CO ice at $20 \mathrm{~K}$}

Figure 3 shows an overview of the experiments performed to derive the $\mathrm{CO}$ photodepletion rate using this method. The substrate coverage of $\mathrm{CO}$ molecules at different VUV fluences are shown. For clarity the experiments covering different thickness regimes are presented with a horizontal offset to be aligned. Previous studies have reported that the top $\sim 4$ to 6 monolayers $(1 \mathrm{ML}=$ $10^{15}$ molecules $\mathrm{cm}^{-2}$ ) play a role in the photodesorption process (Muñoz Caro et al. 2010; Fayolle et al. 2011). Below this substrate coverage, the photodesorption rate drops, and therefore the main set of our experiments is performed in a range where the photodesorption rate is expected to be constant, i.e. for surface coverage above $4 \mathrm{ML}$.

The slope of Fig. 3 represents the $\mathrm{CO}$ photodepletion rate. Combining all experiments, a photodepletion rate of $(1.4 \pm 0.7) \times$ $10^{-3}$ molecules photon $^{-1}$ at $20 \mathrm{~K}$ is obtained. This includes other loss channels, such as photochemistry leading to the formation of $\mathrm{CO}_{2}$. Following the time-of-flight mass spectra we can conclude that typically less than $2 \%$ of the initial $\mathrm{CO}$ is converted to $\mathrm{CO}_{2}$ after being processed by a photon fluence of $5 \times$ $10^{18}$ photons $\mathrm{cm}^{-2}$. This is a minor channel, and negligible given the large relative error $(50 \%)$ in the final photodesorption rate.

\section{Discussion and astrophysical implications}

A correct quantification of the photodesorption rate of species is important as it determines the balance between gas and solid phase molecular reservoirs in astronomical environments such as dense clouds. It is also needed for the interpretation of

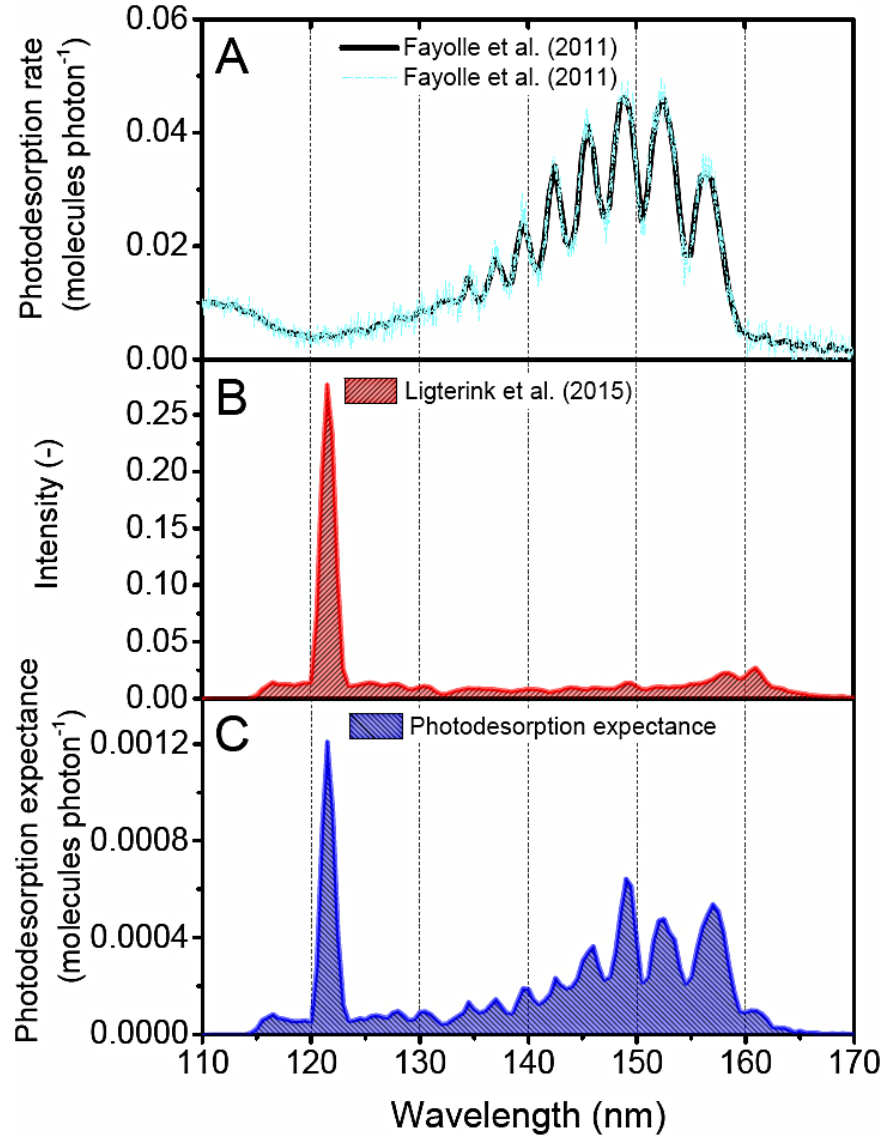

Fig. 4. Panel A): wavelength dependent photodesorption of $\mathrm{CO}$ ice at $18 \mathrm{~K}$ (Fayolle et al. 2011); panel B): normalised VUV spectrum (integrated area $=1$ ) measured with the absolute VUV spectrometer described in Ligterink et al. (2015); panel C): multiplication of panel A) and B) representing the photodesorption expectance based on the VUV spectrum of the $\mathrm{H}_{2}$ lamp. Integration of the curve in panel $\mathbf{C}$ ) provides the estimate of the $\mathrm{CO}$ photodesorption rate at $18 \mathrm{~K}$ based on the spectral characteristics of the applied VUV-lamp.

astronomical observations such as the position of photo-induced snow lines and as input for astrochemical modelling.

\subsection{Overview of the CO photodesorption rates}

With the use of IR spectroscopy and/or mass spectrometry, the photodesorption rate for $\mathrm{CO}$ ice has been measured in a number of studies since 2007 yielding values from $10^{-1}$ to $10^{-3}$ molecules photon ${ }^{-1}$ (Öberg et al. 2007, 2009b; Muñoz Caro et al. 2010, 2016; Chen et al. 2014). Wavelength dependent photodesorption studies of $\mathrm{CO}$ ice at $18 \mathrm{~K}$ using synchrotron radiation have unveiled also information about the underlying process, illustrating that it follows a DIET mechanism, and providing monochromatic photodesorption rates for the first time (Fayolle et al. 2011).

This has strengthened the idea that it is important to know the MW plasma lamp SED characteristics that apply to a specific photo irradiation experiment. Recently, photodesorption experiments have been performed with fully characterised SEDs of the VUV lamp (Chen et al. 2014), but they are still not able to explain the difference in reported photodesorption rates (Ligterink et al. 2015).

Several other causes for the observed deviations have been considered. Different studies have used different methods for the 


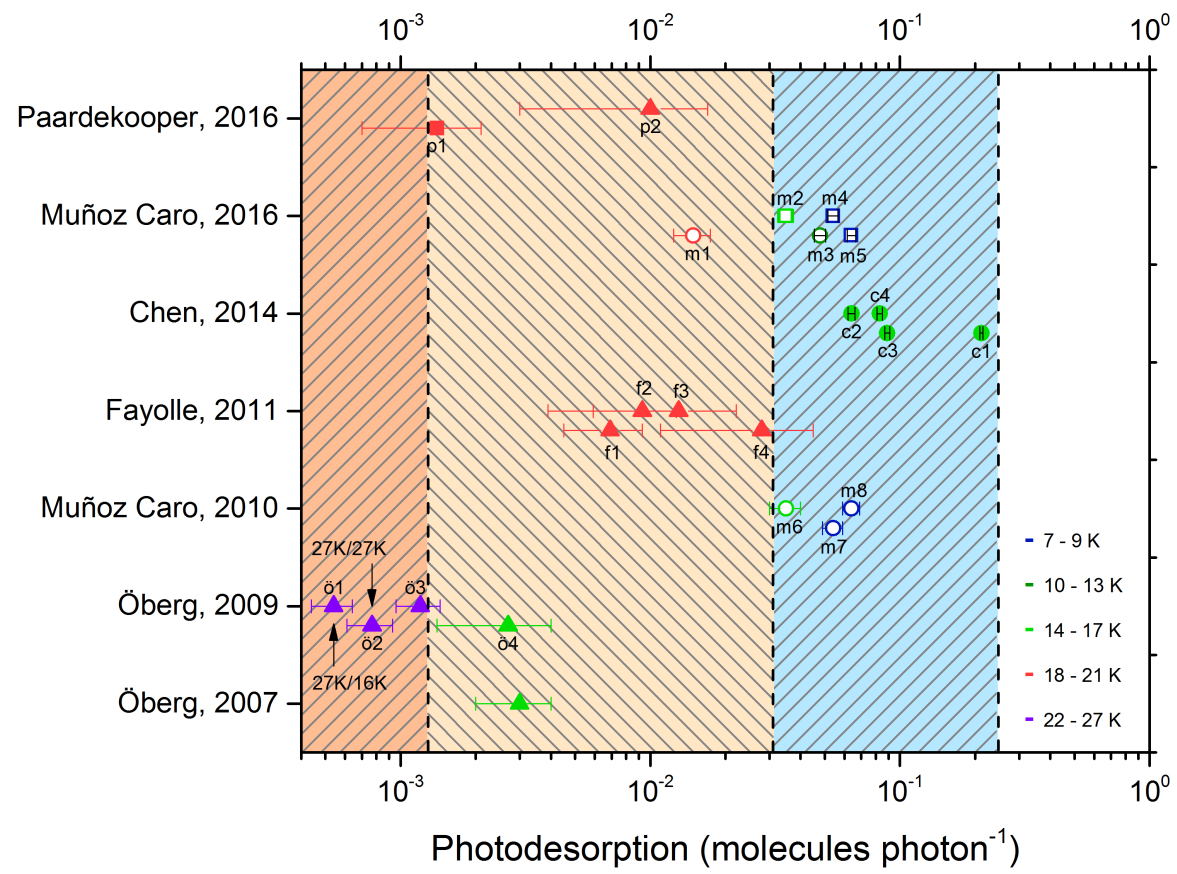

Fig. 5. Overview of all the experimental CO photodesorption studies, the colour code refers to the deposition and photolysis temperature (with the exception of ö1, $27 \mathrm{~K} / 16 \mathrm{~K}$ ). Most studies are performed with a discharge lamp, while Fayolle et al. (2011) use synchrotron irradiation as VUV source $(\mathrm{f} 1=10.2 \mathrm{eV}, \mathrm{f} 2=$ $11.2 \mathrm{eV}, \mathrm{f} 3=9.2 \mathrm{eV}, \mathrm{f} 4=8.2 \mathrm{eV}$ ). Flux calibration; filled symbols: NIST photodiode; empty symbols: actinometry. Detection technique; triangles: RAIRS; circles: FTIR; square: LD TOFMS. The labelling of Chen et al. (2014): c1, c2, c3, and c4 refer to different running conditions of the discharge lamp resulting in different SEDs, while the labelling of the present study refers to the direct measurement (p1) or the indirect measurement (p2) based on the SED of the lamp and wavelength dependence of CO photodesorption from Fayolle et al. (2011) (see Fig. 4).
VUV flux calibration based on either actinometry or NIST calibrated photodiodes, and this may have lead to systematical offsets. It is possible that visible light from the $\mathrm{H}_{2}$ plasma was not fully subtracted, artificially increasing the VUV flux. In addition, it has been observed that the $\mathrm{MgF}_{2}$ windows that are commonly used as a vacuum seal between microwave discharge lamp and UHV set-up, degrade with time, specifically blocking the Ly- $\alpha$ light, i.e. an older window may influence the SED impacting on the ice. These problems concern instrumental issues and, in addition, also the interpretation of data is not straightforward as photoexcitation by VUV photons can induce both photodesorption and/or photochemistry. For CO ice this effect is negligible; the photodepletion signal is very close to the photodesorption value, as discussed before. However, this does not apply to other species, as has recently been illustrated for methanol ice (Bertin et al. 2016). The photodissociation of methanol yields radicals in the ice which can result in photochemistry or, alternatively, the fragments may undergo photodesorption directly after their formation. Finally, the exact ice conditions - ice thickness, ice deposition temperature, and ice morphology - also play a role. For CO ice, Öberg et al. (2009b) observed an ice deposition temperature dependence, as recently confirmed and extended over a wider range ( 7 to $20 \mathrm{~K}$ ) by others, who concluded that this can be linked to the level of $\mathrm{CO}$ dipole orientation within the amorphous state (Muñoz Caro et al. 2010, 2016).

This all makes it hard to decide which photodesorption value is best to use in astrochemical models. This is already true in the case of the largely non-dissociative photodesorption of solid $\mathrm{CO}$; for other species that dissociate upon VUV irradiation the analysis is even more complex.

Thus, for these reasons it is interesting to look for possibilities to link different methods or to use alternative approaches like the one introduced here. In the first case, for example, the recently investigated SED of the $\mathrm{MW} \mathrm{H}_{2}$ lamps (Ligterink et al. 2015) can be linked with the wavelength dependent information obtained by Fayolle et al. (2011). By multiplying the CO photodesorption spectrum (Fig. 4A) and the SED of the lamp used in present study (Fig. 4B), we find a wavelength dependent photodesorption expectance (Fig. 4C). Subsequent integration of this spectrum yields a photodesorption rate, based on the expected SED of the lamp. This results in a photodesorption rate of $(10 \pm 7) \times 10^{-3}$ molecules photon ${ }^{-1}$. The error here is large, as this value is derived by combining the results from two stand-alone experiments. The application of the alternative experimental approach, as introduced in this paper, yields a photodesorption rate for $\mathrm{CO}$ ice at $20 \mathrm{~K}$ of $(1.4 \pm 0.7) \times$ $10^{-3}$ molecules photon ${ }^{-1}$. This value is not too far off from earlier experiments (Öberg et al. 2007, 2009b) and it is in decent agreement with recent molecular dynamics simulations focusing on CO photodesorption; van Hemert et al. (2015) predicted a photodesorption probability of $4 \times 10^{-3}$ molecules photon ${ }^{-1}$, but the value is about one decade off compared to experimental rates reported earlier but typically for conditions that are not fully identical (see Fig. 5).

\section{Conclusion}

This study characterises the photodesorption rate for $\mathrm{CO}$ ice at $20 \mathrm{~K}$, using a new experimental concept. Compared to the established techniques, the method has the extra advantage that the complete ice composition and thickness of the original ice can be derived upon VUV irradiation, which means that both photodesorption and photochemical processes can be quantified.

We have demonstrated the feasibility of this concept on the example of $\mathrm{CO}$ ice at $20 \mathrm{~K}$. We have observed that the $\mathrm{CO}_{2}$ formation channel is minor to negligible. The photodesorption rate of $\mathrm{CO}$ ice at $20 \mathrm{~K}$ is determined as $(1.4 \pm 0.7) \times$ $10^{-3}$ molecules photon $^{-1}$. The $50 \%$ uncertainty of this value is rather high, but not uncommon; most reported photodesorption rates have been determined with errors of the order of $30 \%$. The resulting photodesorption value is in decent agreement with previously reported results for similar conditions. It is clear that for pure $\mathrm{CO}$ ice, photodesorption rates are in the $10^{-3}$ to $10^{-2}$ molecules photon ${ }^{-1}$ range and that at least part of the deviating results can be explained by different experimental settings. For astrochemical models it is particularly important to take into account the deposition temperature; several of the values reported in the literature are measured for values that may be too low to be astrochemically relevant. 
Acknowledgements. This work was supported by NOVA, the Netherlands Research School for Astronomy, a VICI grant from the Netherlands Organisation for Scientific Research (NWO). A. Riedo acknowledges support by the Swiss National Science Foundation (SNSF). Over the years we have collaborated intensely with Jean-Hugues Fillion and Mathieu Bertin and our many discussions on the deviating $\mathrm{CO}$ photodesorption experiments have been an important stimulus for the work presented here. We thank Martijn Witlox for his significant part in the construction of MATRI ${ }^{2}$ CES. We also thank Alan Heays, Catherine Walsh, and Merel 't Hoff for many stimulating discussions.

\section{References}

Andersson, S., \& van Dishoeck, E. F. 2008, A\&A, 491, 907

Andersson, S., Al-Halabi, A., Kroes, G.-J., \& van Dishoeck, E. F. 2006 J. Chem. Phys., 124, 064715

Arasa, C., Andersson, S., Cuppen, H. M., van Dishoeck, E. F., \& Kroes, G.-J. 2010, J. Chem. Phys., 132, 184510

Arasa, C., Andersson, S., Cuppen, H. M., van Dishoeck, E. F., \& Kroes, G. J. 2011, J. Chem. Phys., 134, 164503

Arasa, C., Koning, J., Kroes, G.-J., Walsh, C., \& van Dishoeck, E. F. 2015, A\&A 575, A 121

Bahr, D. A., \& Baragiola, R. A. 2012, ApJ, 761, 36

Baratta, G. A., \& Palumbo, M. E. 1998, J. Opt. Soc. Am., 15, 3076

Bertin, M., Fayolle, E. C., Romanzin, C., et al. 2012, Phys. Chem. Chem. Phys., 14, 9929

Bertin, M., Fayolle, E. C., Romanzin, C., et al. 2013, ApJ, 779, 120

Bertin, M., Romanzin, C., Doronin, M., et al. 2016, ApJ, 817, L12

Bossa, J.-B., Paardekooper, D. M., Isokoski, K., \& Linnartz, H. 2015, Phys. Chem. Chem. Phys., 17, 17346

Bouilloud, M., Fray, N., Bénilan, Y., et al. 2015, MNRAS, 451, 2145

Caselli, P., Keto, E., Bergin, E. A., et al. 2012, ApJ, 759, L37

Chang, Q., \& Herbst, E. 2016, ApJ, 819, 145

Chen, Y.-J., Nuevo, M., Chu, C.-C., et al. 2011, Adv. Space Res., 47, 1633

Chen, Y.-J., Chuang, K.-J., Muñoz Caro, G. M., et al. 2014, ApJ, 781, 15

Cuppen, H. M., Ioppolo, S., Romanzin, C., \& Linnartz, H. 2010, Phys. Chem. Chem. Phys., 12, 12077

DeSimone, A. J., Crowell, V. D., Sherrill, C. D., \& Orlando, T. M. 2013, J. Chem. Phys., 139, 164702

Draine, B. T., \& Salpeter, E. E. 1979, ApJ, 231, 438

Drozdovskaya, M. N., Walsh, C., Visser, R., Harsono, D., \& van Dishoeck, E. F. 2014, MNRAS, 445, 913

Dulieu, F., Congiu, E., Noble, J., et al. 2013, Sci. Rep., 3, 1338

Dzegilenko, F., Herbst, E., \& Uzer, T. 1995, J. Chem. Phys., 102, 2593

Es-sebbar, E.-t., Bénilan, Y., Fray, N., et al. 2015, ApJS, 218, 19

Fayolle, E. C., Bertin, M., Romanzin, C., et al. 2011, ApJ, 739, L36

Fayolle, E. C., Bertin, M., Romanzin, C., et al. 2013, A\&A, 556, A122
Fillion, J.-H., Fayolle, E. C., Michaut, X., et al. 2014, Faraday Discuss., 168, 533 Fuchs, G. W., Cuppen, H. M., Ioppolo, S., et al. 2009, A\&A, 505, 629

Galloway, E. T., \& Herbst, E. 1994, A\&A, 287, 633

Gredel, R., Lepp, S., Dalgarno, A., \& Herbst, E. 1989, ApJ, 347, 289

Hama, T., Yokoyama, M., Yabushita, A., et al. 2010, J. Chem. Phys., 132, 164508

Hartquist, T. W., \& Williams, D. A. 1990, MNRAS, 247, 343

Hollenbach, D., Kaufman, M. J., Bergin, E. A., \& Melnick, G. J. 2009, ApJ, 690, 1497

Ioppolo, S., Öberg, K., \& Linnartz, H. 2014, Laboratory Astrophysics, eds. S. Schlemer, H. Mutschke, T. Giesen, \& C. Jäger (Wiley-VCH Verlag $\mathrm{GmbH} \& \mathrm{Co} . \mathrm{KGaA})$

Leger, A., Jura, M., \& Omont, A. 1985, A\&A, 144, 147

Ligterink, N., Paardekooper, D. M., Chuang, K.-J., et al. 2015, A\&A, 584, A56

Linnartz, H., Ioppolo, S., \& Fedoseev, G. 2015, Int. Rev. Phys. Chem., 34, 205

Martín-Doménech, R., Manzano-Santamaría, J., Muñoz Caro, G. M., et al. 2015, A\&A, 584, A14

Muñoz Caro, G. M., Jiménez-Escobar, A., Martín-Gago, J. Á., et al. 2010, A\&A, 522, A108

Muñoz Caro, G. M., Chen, Y.-J., Aparicio, S., et al. 2016, A\&A, 589, A19

Öberg, K. I. 2016, Chem. Rev., 116, 9631

Öberg, K. I., Fuchs, G. W., Awad, Z., et al. 2007, ApJ, 662, L23

Öberg, K. I., Linnartz, H., Visser, R., \& van Dishoeck, E. F. 2009a, ApJ, 693, 1209

Öberg, K. I., van Dishoeck, E. F., \& Linnartz, H. 2009b, A\&A, 496, 281

Öberg, K. I., Furuya, K., Loomis, R., et al. 2015, ApJ, 810, 112

Paardekooper, D. M., Bossa, J.-B., Isokoski, K., \& Linnartz, H. 2014, Rev. Sci. Instrum., 85

Paardekooper, D. M., Bossa, J.-B., \& Linnartz, H. 2016, A\&A, 592, A67

Piétu, V., Dutrey, A., \& Guilloteau, S. 2007, A\&A, 467, 163

Prasad, S. S., \& Tarafdar, S. P. 1983, ApJ, 267, 603

Roux, J. A., Wood, B. E., Smith, A. M., \& Plyer, R. R. 1980, Infrared optical properties of thin $\mathrm{CO}, \mathrm{NO}, \mathrm{CH} 4, \mathrm{HC} 1, \mathrm{~N} 2 \mathrm{O}, \mathrm{O} 2, \mathrm{AR}$, and air cryofilms, Tech. Rep.

Shen, C. J., Greenberg, J. M., Schutte, W. A., \& van Dishoeck, E. F. 2004, A\&A, 415, 203

van Hemert, M. C., Takahashi, J., \& van Dishoeck, E. F. 2015, J. Phys. Chem. A, 119,6354

Vastel, C., Ceccarelli, C., Lefloch, B., \& Bachiller, R. 2014, ApJ, 795, L2

Walsh, C., Nomura, H., \& van Dishoeck, E. 2015, A\&A, 582, A88

Watanabe, N., \& Kouchi, A. 2002, ApJ, 571, L173

Westley, M. S., Baragiola, R. A., Johnson, R. E., \& Baratta, G. A. 1995a, Nature, 373,405

Westley, M. S., Baragiola, R. A., Johnson, R. E., \& Baratta, G. A. 1995b, Planet. Space Sci., 43, 1311

Willacy, K., \& Langer, W. D. 2000, ApJ, 544, 903

Yuan, C., \& Yates, J. T. 2013, J. Chem. Phys., 138, 154302

Zhen, J., \& Linnartz, H. 2014, MNRAS, 437, 3190 